\title{
Spin observables in deuteron-proton radiative capture at intermediate energies
}

\author{
A.A. Mehmandoost-Khajeh-Dad a,b H.R. Amir-Ahmadi a , J.C.S. Bacelar ${ }^{\text {a }}$, \\ A.M. van den Berg ${ }^{\text {a }}$, R. Castelijns ${ }^{\text {a }}$, A. Deltuva ${ }^{\text {c, },}$, E.D. van Garderen ${ }^{a}$, W. Glöckle ${ }^{\text {, }}$, \\ J. Golak ${ }^{\mathrm{e}}$, N. Kalantar-Nayestanaki ${ }^{\mathrm{a}}, \mathrm{H}$. Kamada ${ }^{\mathrm{f}}$, M. Kiš ${ }^{\mathrm{a}, 2}$, \\ R. Koohi-Fayegh-Dehkordi ${ }^{\text {b }}$ H. Löhner ${ }^{\text {a }}$, M. Mahjour-Shafiei ${ }^{\text {a, }}$, H. Mardanpour ${ }^{\text {a }}$, \\ J.G. Messchendorp ${ }^{a}$, A. $\operatorname{Nogga}^{\mathrm{g}}$, P. Sauer ${ }^{\mathrm{c}}$, S.V. Shende ${ }^{\mathrm{a}}$, R. Skibinski ${ }^{\mathrm{e}}, \mathrm{H}$. Witała ${ }^{\mathrm{e}}$, \\ H.J. Wörtche ${ }^{\text {a }}$ \\ ${ }^{a}$ Kernfysisch Versneller Instituut (KVI), Groningen, The Netherlands \\ ${ }^{\mathrm{b}}$ Mashad Ferdowsi University, Mashad, Iran \\ ${ }^{\mathrm{c}}$ Institute for Theoretical Physics, University of Hannover, Hannover, Germany \\ ${ }^{\mathrm{d}}$ Institüt für Theoretische Physik II, Ruhr-Universität Bochum, Bochum, Germany \\ ${ }^{\mathrm{e}}$ M. Smoluchowski Institute of Physics, Jagiellonian University, Kraków, Poland \\ ${ }^{\mathrm{f}}$ Department of Physics, Faculty of Engineering, Kyushu Institute of Technology, 1-1 Sensuicho, Tobata, Kitakyushu 804-8550, Japan \\ $\mathrm{g}$ Institute for Nuclear Theory, University of Washington, Seattle, WA, USA
}

Received 18 January 2005; received in revised form 11 April 2005; accepted 29 April 2005

Available online 11 May 2005

Editor: V. Metag

\begin{abstract}
A radiative deuteron-proton capture experiment was carried out at KVI using polarized-deuteron beams at incident energies of 55, 66.5, and $90 \mathrm{MeV} /$ nucleon. Vector and tensor-analyzing powers were obtained for a large angular range. The results are interpreted with the help of Faddeev calculations, which are based on modern two- and three-nucleon potentials. Our data are described well by the calculations, and disagree significantly with the observed tensor anomaly at RCNP.
\end{abstract}

○ 2005 Elsevier B.V. All rights reserved.

E-mail address: messchendorp@kvi.nl (J.G. Messchendorp).

1 Present address: Centro de Fisica Nuclear da Universidade de Lisboa, Lisboa, Portugal.

2 Present address: Rudjer Boskovic Institute, Zagreb, Croatia.

3 Present address: Tehran University, Tehran, Iran. 
The structure of nuclei and the dynamics of reactions are described by the strong nuclear force governing the nucleon-nucleon interaction [1]. The longestrange two-nucleon force (2NF) is due to the exchange of a pion [2], an idea that goes back to the work of Yukawa in 1935. At present, 2NF models exist which provide an excellent description of the high-quality data base of proton-proton and neutron-proton scattering and of the properties of the deuteron. For heavier nuclei, Green's function Monte Carlo calculations employing 2NFs clearly underestimate the experimental binding energies [3], and therefore show that 2NF are not sufficient to describe the three-nucleon system and heavier systems accurately.

In the last decades, our understanding of the threenucleon system has improved significantly. Highprecision data at intermediate energies in $\mathrm{Nd}$ elastic scattering [4-6] for a large energy interval together with rigorous Faddeev calculations [7] for the threenucleon system have constrained phenomenological three-nucleon forces (3NF). These studies are supported by calculations based on $\chi \mathrm{PT}$ at lower energies, which are expected to provide model-independent predictions for the complete structure of the $3 \mathrm{NF}$ [8-11] in the near future.

The radiative deuteron-proton capture reaction, $p+d \rightarrow{ }^{3} \mathrm{He}+\gamma$, is an interesting channel since it involves a large momentum transfer and therefore probes high-momentum components of the wave functions involved in the matrix element. In addition, the coupling with a photon makes this reaction sensitive to electromagnetic currents involved in the three-nucleon system. These aspects make the radiative capture process a unique tool to extend the above described three-nucleon force studies.

In the last few years, the interest in the radiative $N d$ capture channel has increased. This is partly due to the presently available theoretical techniques which solve the three-nucleon system rigorously. In contrast to the elastic $N d$ scattering data, however, the experimental data base on radiative $N d$ capture is much poorer. In particular, in the intermediate energy range ( 50-200 MeV/nucleon), below the pion-production threshold, the available data [12-15] are scarce and in general lack precision or completeness in angular coverage and the number of observables.

Recently, a precision deuteron-proton radiative capture experiment $[16,17]$ at RCNP was conducted using a vector and tensor-polarized deuteron beam impinging on a proton target at an incident deuteron energy of $100 \mathrm{MeV} /$ nucleon. Interestingly, the preliminary results on tensor-analyzing powers $[16,17]$ show large discrepancies with present-day calculations. These deviations were found to be larger than a factor three for $A_{x x}$ in comparison with several different model approaches. As a result, the authors speculated about possible existence of new forces or new mechanisms that are sensitive to tensor observables. A confirmation of this intriguing tensor anomaly in $p d$-radiative capture is clearly needed. In addition, a study of the energy dependence is necessary in order to understand the origin of these discrepancies.

In this Letter, deuteron-proton radiative capture data on vector and tensor-analyzing powers obtained at KVI are presented along with a comparison with two theoretical approaches. The first calculation by the Bochum-Cracow group $[18,19]$ is a Faddeev calculation with the AV18 2NF and an additional phenomenological Urbana IX $3 \mathrm{NF}$ as input. The coupling with a photon is described via two different approaches. The first approach supplements the single-nucleon current operator by exchange currents which take explicitly into account $\pi$ - and $\rho$-like meson-exchange contributions. Alternatively, the meson-exchange currents are included using the extended Siegert theorem. In this form, electric and magnetic multipoles are kept to very high orders for the one-body operator. As a consequence of the Siegert approximation, only manybody currents in the electric multipoles are accounted for. The second calculation is from the Hannover theory group [20], which describes the process using the purely nucleonic charge-dependent CD-Bonn potential and its coupled-channel extension CD-Bonn $+\Delta$. Within this approach, the $\Delta$-isobar excitation mediates an effective 3NF with prominent Fujita-Miyazawa and Illinois ring type contributions. These contribu- 


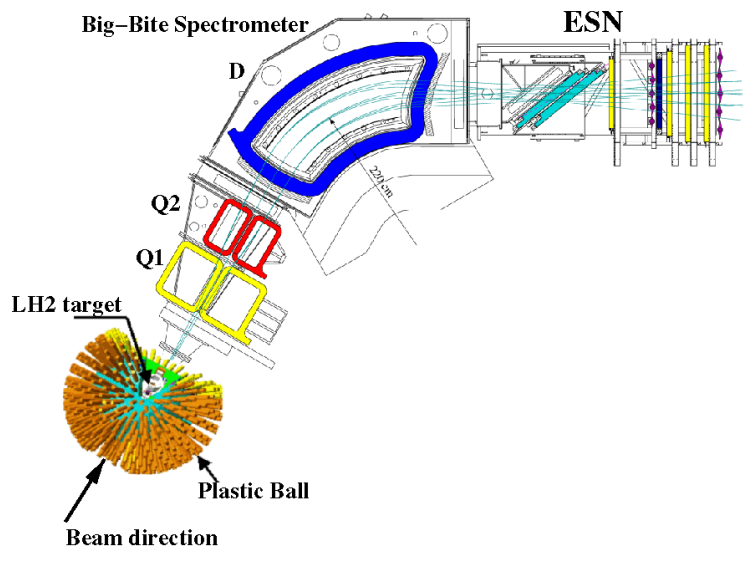

Fig. 1. A sketch of the experimental setup at KVI, which measured the deuteron-proton radiative capture reaction. The Big-Bite spectrometer (BBS) was employed to detect the ${ }^{3} \mathrm{He}$ at forward angles. The photon was detected between $50^{\circ}$ and $160^{\circ}$ using the Plastic Ball detector. A cryogenic proton target was placed at the center of the Plastic Ball.

tions are based on the exchange of $\pi, \rho, \omega$, and $\sigma$ mesons and are mutually consistent. The electromagnetic current in the Hannover approach has onebaryon and two-baryon contributions and couples to nucleonic and $\Delta$-isobar channels. Therefore, the $\Delta$ isobar generates consistently effective two- and threenucleon currents in addition to a $3 \mathrm{NF}$.

The experiment was carried out in autumn of 2003 at KVI, The Netherlands. Beams of vector and tensorpolarized deuterons were produced in an atomicbeam-type ion source (POLIS) and accelerated with the superconducting cyclotron, AGOR, up to 55, 66.5, and $90 \mathrm{MeV} /$ nucleon. The beam with an intensity of $\approx 0.5 \mathrm{nA}$ impinged on a $4.5 \mathrm{~mm}$ thick liquid-hydrogen target. The ${ }^{3} \mathrm{He}$ particle and the photon were detected using a coincidence setup between the Big-Bite spectrometer (BBS) and the Plastic Ball detector (PB), respectively. The setup is depicted in Fig. 1. The magnetic spectrometer BBS [21], with an angular acceptance of $\approx 3.8^{\circ}$, with its Euro-Supernova focal-plane detection system (ESN) [22] was placed at various angles between $1.7^{\circ}$ and $3.5^{\circ}$ for different energies to cover as large a center-of-mass angular range as possible. With this detector, nearly the complete ${ }^{3} \mathrm{He}$ phase space is covered. The energy and angle resolutions were dominated by straggling in the target. However, the two-body reconstruction was enough to identify the events adequately (see Fig. 2). The

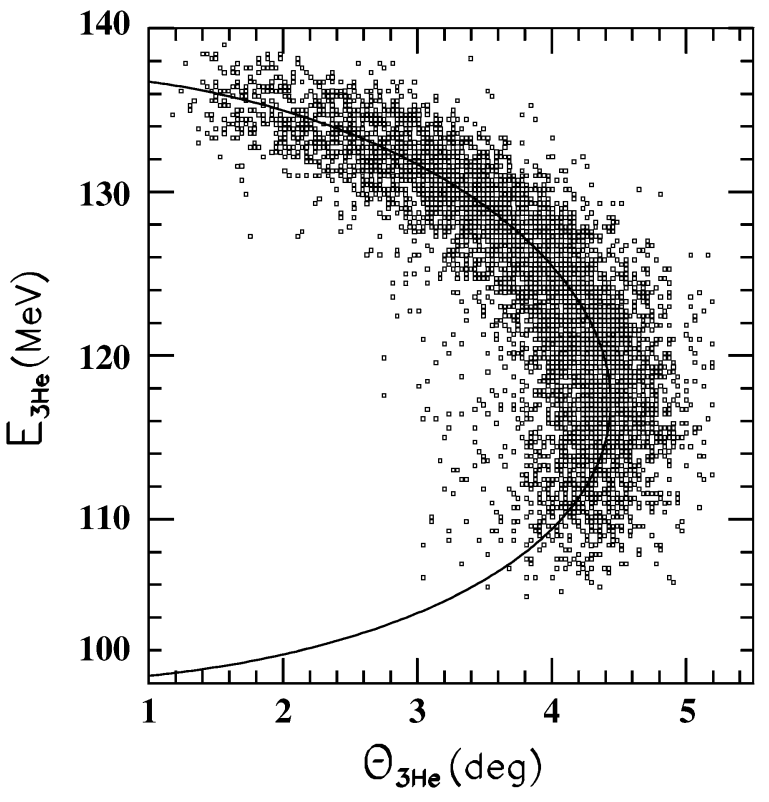

Fig. 2. The measured energy of ${ }^{3} \mathrm{He}$ versus its scattering angle measured by the BBS placed at a scattering angle of $3.5^{\circ}$. The data are obtained with a $90 \mathrm{MeV} /$ nucleon deuteron beam. The solid line represents the expected kinematical dependence of the deuteron-proton radiative capture process. The width of the band is due to energy straggling in the target.

PB detector [23] was equipped with $\approx 500 \Delta E-E$ phoswich modules covering photon scattering angles between $50^{\circ}$ and $160^{\circ}$ with complete azimuthal acceptance. Each module contains a $4 \mathrm{~mm}$ thick $\mathrm{CaF}_{2}$ layer (slow component) glued on to a $356 \mathrm{~mm}$ thick scintillator (fast component), which allows to discriminate photons, leptons, and protons from each other. The PB detector measures the scattering angle of the photon with a resolution of $6^{\circ}$ and with an efficiency of $\approx 50 \%$.

Fig. 2 demonstrates the quality of the ${ }^{3} \mathrm{He}$ analysis with the BBS detection system. Here, the measured energy of the ${ }^{3} \mathrm{He}$ is plotted against its scattering angle obtained from the BBS for an incident deuteron beam energy of $90 \mathrm{MeV} /$ nucleon. The solid line represents the expected kinematical correlation for the radiative capture reaction. A coincidence with a photon detected by the PB is required in this plot. The lower part of the kinematical loci is not covered experimentally due to a lack of photon detectors at scattering angles below $50^{\circ}$ in the laboratory system. The data coincide with the expected kinematical correlation. Background chan- 
nels, like radiative break-up, will not follow such a behavior. This demonstrates that the reaction of interest can be identified unambiguously.

Vector and tensor-analyzing powers in the deuteronproton radiative capture process were obtained by employing a beam of polarized deuterons. Five different polarization states were provided by the ion source with theoretical polarization values of $\left(\mathrm{p}_{Z}, \mathrm{p}_{Z Z}\right)=$ $(0,0),(2 / 3,0),(-2 / 3,0),(0,1),(0,-2)$. In this notation, $\mathrm{p}_{Z}$ and $\mathrm{p}_{Z Z}$ represent the vector and tensor polarizations of the deuteron ion beam at the source. The beam polarization with a typical value of $70-80 \%$ of the theoretical value was monitored regularly using the in-beam polarimeter (IBP) [24]. This polarimeter uses a measurement of the azimuthal asymmetry in the elastic $\vec{d}+p$ reaction at a centerof-mass angle of $101^{\circ}$. To determine an absolute polarization, a knowledge of the tensor and vector analyzing powers of the elastic $\vec{d}+p$ reaction is required. These values were obtained from a fit through published data at energies ranging from 30 to $100 \mathrm{MeV} /$ nucleon [5,25,26]. An interpolation yields vector and tensor analyzing powers of $i T_{11}=-0.39 \pm$ $0.04, T_{22}=-0.128 \pm 0.020, T_{20}=-0.255 \pm 0.025$ for an energy of $55 \mathrm{MeV} /$ nucleon, $i T_{11}=-0.44 \pm$ $0.04, T_{22}=-0.161 \pm 0.020, T_{20}=-0.273 \pm 0.023$ for $66.5 \mathrm{MeV} /$ nucleon, and $i T_{11}=-0.43 \pm 0.04$, $T_{22}=-0.214 \pm 0.020, T_{20}=-0.237 \pm 0.023$ for $90 \mathrm{MeV} /$ nucleon.

In this Letter, we present a measurement of vector $\left(A_{y}(d)\right)$ and tensor $\left(A_{y y}, A_{z z}\right)$ analyzing powers of the $\vec{d}+p \rightarrow{ }^{3} \mathrm{He}+\gamma$ reaction. These observables were extracted by making use of the dependence on the azimuthal angle, $\phi$, of the reaction rate, $I(\theta, \phi)$, according to [27]

$$
\begin{aligned}
\frac{I(\theta, \phi)}{I_{0}(\theta)}= & 1+\frac{3}{2} \mathrm{p}_{Z} A_{y}(\theta) \cos \phi-\frac{1}{2} \mathrm{p}_{Z Z} A_{z z}(\theta) \sin ^{2} \phi \\
& +\frac{1}{2} \mathrm{p}_{z Z} A_{y y}(\theta) \cos 2 \phi,
\end{aligned}
$$

where $I_{0}(\theta)$ is the reaction rate for an unpolarized beam and $\theta$ is the polar angle of the $\gamma-p$ system in the center-of-mass. Note that at KVI the polarization vector is perpendicular to the beam direction. Exploiting the above equation, the vector analyzing power, $A_{y}(d)$, is obtained from the reaction rates for ion-source states $\left(\mathrm{p}_{Z}, \mathrm{p}_{Z Z}\right)=(2 / 3,0)\left(N^{+}\right)$and $(-2 / 3,0)\left(N^{-}\right)$integrating $\phi$ from $-55^{\circ}$ to $55^{\circ}$ for a beam energy of $90 \mathrm{MeV} /$ nucleon and from $-65^{\circ}$ to $65^{\circ}$ for beam energies of 55 and $66.5 \mathrm{MeV} /$ nucleon, according to

$$
A_{y}(d)=-\frac{2}{3} \frac{N^{+}-N^{-}}{\mathrm{p}_{Z}^{-} N^{+}-\mathrm{p}_{Z}^{+} N^{-}},
$$

where $\mathrm{p}_{Z}^{+}$is the measured vector polarization for the $(2 / 3,0)$ spin mode and $\mathrm{p}_{Z}^{-}$is the polarization for the $(-2 / 3,0)$ spin mode. The azimuthal angle, $\phi$, is obtained from the PB. Corrections due to variations in the photon-detection efficiency are properly taken into account by measuring $\phi$ distributions using the unpolarized data of the radiative-capture reaction. Similarly, the tensor-analyzing power, $A_{z z}$, is deduced using the states $\left(\mathrm{p}_{Z}, \mathrm{p}_{Z Z}\right)=(0,1)$ and $(0,-2)$ integrating $\phi$ from $35^{\circ}$ to $55^{\circ}$ (and from $-35^{\circ}$ to $-55^{\circ}$ ) for a beam energy of $90 \mathrm{MeV} /$ nucleon and integrating $\phi$ from $25^{\circ}$ to $65^{\circ}$ (and from $-25^{\circ}$ to $-65^{\circ}$ ) for a beam energy of $66.5 \mathrm{MeV} /$ nucleon. According to Eq. (1), this integration should cancel the contribution of $A_{y y}$ to the cross section. Remaining contributions of $A_{y y}$ due to variations in the photon-detection efficiency in $\phi$ are properly corrected for. The tensoranalyzing power, $A_{y y}$, is obtained by integrating, for the same ion states, over the azimuthal angle $\phi$ from $-15^{\circ}$ to $15^{\circ}$. Also here, non-vanishing contributions of the term $\frac{1}{2} \mathrm{p}_{Z Z} A_{z z} \sin ^{2} \phi$ in Eq. (1) due to a finite $\phi$ integration are small $(\sim 2 \%)$ and corrected for.

Fig. 3 shows the results of the deuteron-proton radiative capture experiment in comparison with the calculation by the Bochum-Cracow group $[18,19]$. Data for $A_{y}(d), A_{y y}$, and $A_{z z}$ are presented as a function of the $\gamma-p$ center-of-mass angle for three different incident deuteron energies of 55, 66.5, and $90 \mathrm{MeV} /$ nucleon. Only statistical uncertainties are indicated by the error bars. The systematic uncertainty due to the error in the beam polarization is estimated to be less than $6 \%$. The dotted lines are the result of the Faddeev calculation for which the AV18 2NF is used as input. Meson-exchange currents are included using the Siegert approximation. The dot-dashed lines correspond to the same model and including the Urbana-IX $3 N F$. An explicit inclusion of $\pi$ and $\rho$ like mesonexchange contributions are represented by the dashed and solid lines. For the solid lines, the Urbana-IX 3NF was included, whereas the dashed lines only take into account the AV18 2NF. The data for $A_{y}(d)$ clearly dis- 


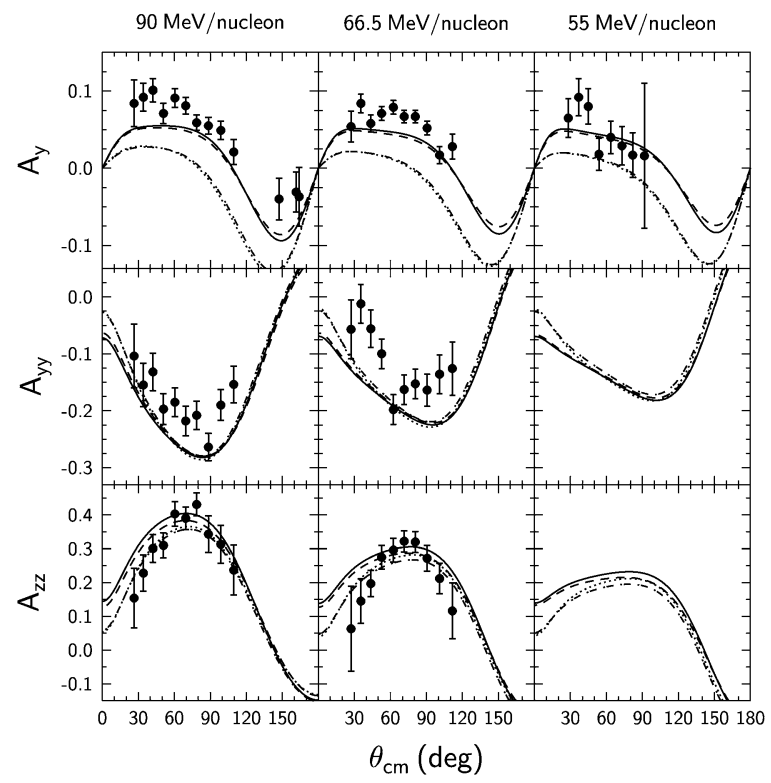

Fig. 3. Polarization data for the deuteron-proton radiative capture reaction are compared to Faddeev calculations by the Bochum-Cracow theory group. The data are shown as filled circles with the statistical uncertainty indicated by error bar. The dotted and dot-dashed lines (which can hardly be distinguished) represent the results of the calculation using the Siegert approximation with the AV18 2NF as input and with the additional inclusion of the Urbana-IX $3 \mathrm{NF}$, respectively. The dashed (2NF) and solid $(2 \mathrm{NF}+3 \mathrm{NF})$ lines are similar calculations for which meson-exchange currents are calculated using explicit $\pi$ and $\rho$ exchanges.

agree with the calculation in which meson-exchange contributions are constructed using the Siegert approximation. This might point to large magnetic contributions which are not properly included for many-body currents. The approximation with an explicit inclusion of $\pi$ and $\rho$ exchange agrees well with our data. The effect of the $3 \mathrm{NF}$ is small within the framework of this approximation. At present, no experiment will have sufficient sensitivity to prefer this calculation with or without the inclusion of a $3 \mathrm{NF}$.

Fig. 4 compares the same data as in Fig. 3 with the predictions of the Hannover group [20], based on the purely nucleonic CD-Bonn potential and its coupled-channel extension, CD Bonn $+\Delta$, allowing for a single excitation of a nucleon to a $\Delta$ isobar. The $\Delta$ mediates 3 NFs and generates effective two- and three-nucleon currents in addition to irreducible oneand two-baryon contributions as described in detail in

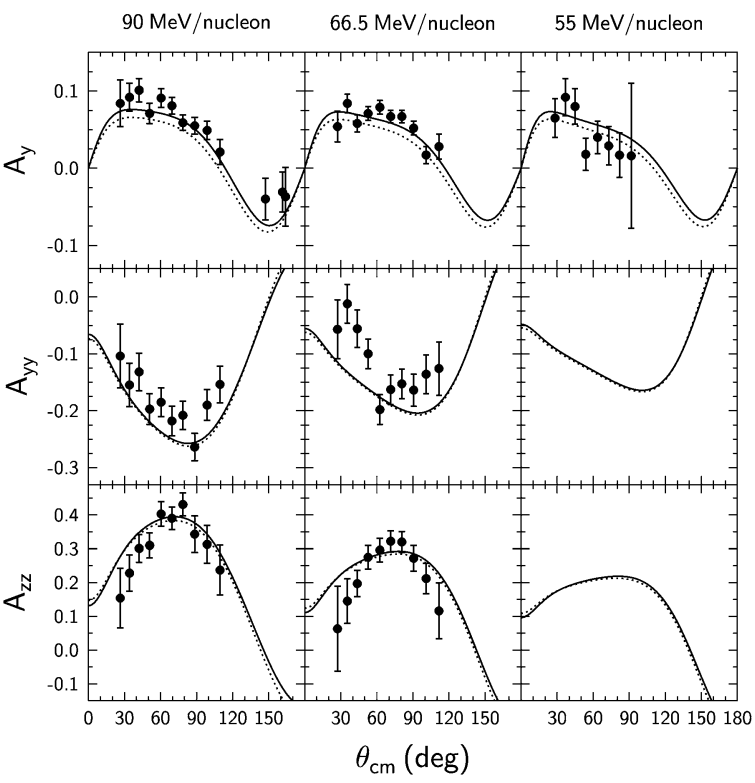

Fig. 4. The same data as shown in Fig. 3 are compared with the predictions of the Hannover group. The dashed line represents the calculation based on the CD-Bonn potential, whereas the solid line includes in addition contributions from the $\Delta$ isobar.

Ref. [20]. Note that the Hannover calculation agrees reasonably well with our data for all polarization observables and all energies. The effect of the $\Delta$ isobar is small at these energies and for these observables.

The tensor-analyzing powers $A_{z z}$ and $A_{y y}$ hardly depend on the choice of approximation. The predictions from the Bochum-Cracow group are similar to those of the Hannover group for these observables. Since the tensor-analyzing power $A_{x x}$ is related to $A_{y y}$ and $A_{z z}$, via $A_{x x}+A_{y y}+A_{z z}=0$, we conclude that also this observable is rather well predicted by all models including MECs. Surprisingly, a recent experiment conducted at RCNP with a $100 \mathrm{MeV} /$ nucleon incident deuteron beam showed large deviations for $A_{x x}$ in comparison with similar model predictions [16, 17]. Our data taken at an energy of $90 \mathrm{MeV} /$ nucleon clearly do not show such large discrepancies, and therefore contradict the preliminary data of RCNP. Also at lower energies, no anomaly is observed for the tensor-analyzing powers in the deuteron-proton radiative capture process.

In summary, this Letter presents data on vector and tensor-analyzing powers in the deuteron-proton radiative capture process. The data were taken at KVI with 
the Big-Bite spectrometer and the almost $-4 \pi$ Plastic Ball detection systems which measure the momentum vectors of the ${ }^{3} \mathrm{He}$ and $\gamma$, respectively. A polarized deuteron beam was employed at incident energies of $55,66.5$, and $90 \mathrm{MeV} /$ nucleon. The reaction rate dependence on the azimuthal angle together with different combinations for the polarization states $\left(\mathrm{p}_{Z}, \mathrm{p}_{Z Z}\right)$ were used to extract $A_{y}(d), A_{y y}$, and $A_{z z}$ for a large angular range. The results are interpreted using Faddeev calculations by the Bochum-Cracow group and by the Hannover group. In general, our results agree reasonably well with predictions by the Hannover group and the predictions by the Bochum-Cracow group in case an explicit $\pi$ and $\rho$ exchange is used. The large discrepancy of our data with the calculation by the Bochum-Cracow group using the Siegert approximation demonstrates the sensitivity to the treatment of the electromagnetic currents in the radiativecapture reaction. In particular, the vector-analyzing powers are rather sensitive to MECs making them a good testing ground for the details of exchange currents. The calculations and experimental results presented in this Letter indicate that the effect of a phenomenological three-nucleon force and the contribution of the $\Delta$ isobar are, at these energies, small. Therefore, these observables are ideally suited to test electromagnetic currents and form factors in a threenucleon system. The study presented in this Letter was partly motivated by a recent observation of a large discrepancy between measured tensor-analyzing powers taken at RCNP and predictions by various modern calculations. Our data taken at a similar energy do not show such a discrepancy and therefore disagree with the preliminary RCNP data.

\section{Acknowledgements}

The authors acknowledge the work by the cyclotron and ion-source groups at KVI for delivering the high-quality beam used in these measurements and thank Muhsin Harakeh for a careful proofreading of this Letter. This work is part of the research program of the "Stichting voor Fundamenteel Onderzoek der Materie" (FOM) with financial support from the "Nederlandse Organisatie voor Wetenschappelijk Onderzoek" (NWO). The work of the Cracow-Bochum group was supported by the Polish Committee for Scientific Research under Grants No. 2P03B0825. The numerical calculations have been performed on the Cray SV1 of the NIC in Jülich, Germany.

\section{References}

[1] L.M. Brown, H. Rechenberg, The Origin of the Concept of Nuclear Force, IOP, Bristol, 1996.

[2] V. Stoks, et al., Phys. Rev. C 47 (1993) 512.

[3] R.B. Wiringa, S.C. Pieper, Phys. Rev. Lett. 89 (2002) 182501.

[4] K. Ermisch, et al., Phys. Rev. Lett. 86 (2001) 5862.

[5] K. Sekiguchi, et al., Phys. Rev. C 65 (2002) 034003.

[6] K. Ermisch, et al., Phys. Rev. C 68 (2003) 051001.

[7] W. Glöckle, et al., Phys. Rep. 274 (1996) 107.

[8] C. Ordóñez, U. van Kolck, Phys. Lett. B 291 (1992) 459.

[9] U. van Kolck, Phys. Rev. C 49 (1994) 2932.

[10] E. Epelbaum, et al., Nucl. Phys. A 637 (1998) 107.

[11] J.L. Friar, et al., Phys. Rev. C 59 (1999) 53.

[12] J.M. Cameron, et al., Nucl. Phys. A 424 (1984) 549.

[13] M.J. Pickar, et al., Phys. Rev. C 35 (1987) 37.

[14] R. Johansson, et al., Nucl. Phys. A 641 (1998) 389.

[15] J.G. Messchendorp, et al., Phys. Lett. B 481 (2000) 171.

[16] K. Sagara, et al., in: Proceedings of the Seventeenth International IUPAP Conference on Few-Body Problems in Physics, Elsevier, Amsterdam, 2003, p. S149.

[17] T. Yagita, et al., Mod. Phys. Lett. A 18 (2003) 322.

[18] R. Skibinski, et al., Phys. Rev. C 67 (2003) 054001.

[19] J. Golak, et al., Phys. Rev. C 62 (2000) 054005.

[20] A. Deltuva, et al., Phys. Rev. C 69 (2004) 034004.

[21] A.M. van den Berg, Nucl. Instrum. Methods Phys. Res. B 99 (1995) 637.

[22] H.J. Wörtche, Nucl. Phys. A 687 (2001) 321.

[23] A. Baden, et al., Nucl. Instrum. Methods 203 (1982) 189.

[24] R. Bieber, et al., Nucl. Instrum. Methods Phys. Res. A 457 (2001) 12.

[25] K. Hatanaka, et al., Nucl. Phys. A 426 (1984) 77.

[26] H. Witała, et al., Few Body Systems 15 (1993) 67.

[27] G.G. Ohlsen, Rep. Prog. Phys. 35 (1972) 717. 